\title{
CETOSE BOVINA - REVISÃO DA LITERATURA
}

\author{
BATISTA, Flávio Cristino Carmo ${ }^{1}$ \\ D'AURIA, Eliana ${ }^{2}$ \\ PALAZZO, Elzylene Léga²
}

RESUMO: O período de transição corresponde a três semanas antes e após o parto e é um momento de grandes desafios fisiológicos para o gado leiteiro como também para pecuaristas e veterinários. Diversos distúrbios metabólicos podem ocorrer entre o final da gestação e o pico de lactação de vacas leiteiras, entre eles, a cetose. Esta revisão da literatura aborda como ocorre a doença, o diagnóstico, o tratamento e a prevenção da cetose em bovinos leiteiros, a fim de explicar os prejuízos econômicos e comprometimento da saúde do animal que esta afecção provoca.

Palavras-chaves: Glicemia. Período de transição. Corpos cetônicos. Vacas Leiteiras.

\section{CATTLE KETOSIS - A REVIEW}

SUMMARY: The transition period is period between three weeks prior calving and three after this is a moment of huge physiological challenges for dairy cows as well for dairy producers and veterinarians. Many metabolic disorders can occur between late gestation and lactation peak of milk among them the ketosis. This bibliography review describes how ketosis occurs, the diagnostics, treatments and prevention in dairy cows, in order to explain the economics losses and compromising of animal health that this disease brings to the herd.

Keywords: Glycemia. Transition Period. Ketone Bodies. Dairy Cows.

\section{INTRODUÇÃO}

O período de transição em vacas leiteiras é definido três semanas antes e após o parto, sendo caracterizado por significativas mudanças fisiológicas e imunológicas devido ao parto e início da lactação. Este é um período crítico para os animais no qual ocorre alta incidência de doenças metabólicas e infecciosas (VICENTE et al., 2014).

A cetose é uma doença metabólica que ocorre no pós-parto em vacas de alta produção por distúrbio multifatorial do metabolismo energético. A cetose pode ocorrer na forma clínica ou subclínica causando redução da produção leiteira, diminuição do teor de gordura no leite, redução

\footnotetext{
1 Acadêmico do curso de Medicina Veterinária da Faculdade Dr. Francisco Maeda, Fundação Educacional de Ituverava. Rodovia Jeronimo Nunes Macedo, Km 01, 14500-000 Ituverava-SP. E-mail: fb.vet86@gmail.com

2 Professora da Faculdade Dr. Francisco Maeda, Fundação Educacional de Ituverava. Rodovia Jeronimo Nunes Macedo, Km 01, 14500-000 Ituverava-SP.
} 
no desempenho reprodutivo e aumento no risco de manifestar deslocamento de abomaso (DIVERS; PEEK, 2008).

A cetose é causada devido ao balanço energético negativo (BEN) em vacas de alta produção leiteira. O máximo consumo de matéria seca (CMS) no pós-parto não ocorre antes das 10 semanas pós-parto, mas, como o pico de lactação acontece entre quatro e seis semanas de lactação, a ingestão de energia não acompanha a demanda. Devido ao BEN, os níveis de glicose e insulina sanguíneos caem drasticamente, em resposta, o animal mobiliza tecido adiposo o que aumenta os níveis séricos de ácidos graxos não-esterificados (AGNE). Os AGNE são sintetizados no fígado e produzem os corpos cetônicos acetoacetato, acetona e beta-hidroxibutirato (BHBA) (RADOSTISTS et al., 2000).

A cetose é diagnosticada pelos sinais clínicos e mensuração de corpos cetônicos no leite, sangue e urina. Segundo Oetzel (2004), vacas com BHBA entre 1,2 a 2,9 mmol/L são consideradas com cetose subclínica e vacas com BHBA maior que $3 \mathrm{mmol} / \mathrm{L}$ são classificadas com cetose clínica. Geralmente, o aumento de corpos cetônicos no sangue é acompanhado de hipoglicemia.

A mensuração de BHBA no soro ou plasma é considerado um ótimo método de diagnóstico para cetose. Entretanto, o diagnóstico realizado com o uso de um sistema eletrônico que mensura os níveis de BHBA e glicose sanguíneos possui grande acurácia (DUFFIELD, 2000; HERDT, 2000). Descrito por Araújo (2013), o medidor digital de BHBA indicado para uso humano, é também indicado para uso em bovinos leiteiros, sendo facilmente adquirido no mercado.

\section{CONTEÚDO}

O metabolismo energético dos ruminantes é regulado pela manutenção dos níveis adequados de glicose no sangue (RADOSTITS et al., 2000). Segundo Bacila (2003), a taxa de glicose sanguínea em um bovino adulto é entre 45 e $75 \mathrm{mg} / \mathrm{dL}$. Devido a alta taxa de fermentação dos carboidratos no rúmen, pouca glicose é absorvida diretamente da dieta (DRACKLEY et al., 2005). Deste modo, a maior parte da glicose é suprida pela gliconeogênese hepática. Os ácidos graxos voláteis (AGV) provenientes da fermentação de carboidratos no rúmen e os aminoácidos são os principais percursores da gliconeogênese (RADOSTITS et al., 2000). Os AGV são absorvidos pela parede ruminal e alcançam o fígado, pelo sistema porta hepático onde serão substratos para a glicogeneogêse (BACILA, 2003). Os principais AGV são o acetato, o 
propionato e butirato produzidos em uma proporção de 70\%, 20\% e 10\% respectivamente, em animais que recebem dietas ricas em fibra, e 60\%, 30\% e 10\%, em animais que recebem dietas ricas em grãos (SOUZA, 2003).

O acetato é principalmente utilizado na síntese de gorduras ou secretado como gordura do leite; o butirato é metabolizado em acetil-CoA podendo ser oxidado formando corpos cetônicos; e o propionato é o principal percursor de glicose nos ruminantes penetrando diretamente no ciclo de Krebs. O fígado é o responsável pela gliconeogênese a partir do propionato. O tecido adiposo é a principal forma de armazenamento de energia para os animais. A gordura não pode ser convertida em glicose, entretanto, os ácidos graxos estocados em forma de gordura podem ser convertidos em corpos cetônicos (CUNNINGHAM, 2004). O metabolismo das gorduras e do butirato produzem os corpos cetônicos BHBA, acetoacetato e acetona (SOUZA, 2003). Os corpos cetônicos são produtos fisiológicos do metabolismo dos ruminantes importantes na manutenção da homeostase, porém, um aumento exacerbado na sua produção pode desencadear um quadro patológico (CUNNINGHAM, 2004).

O período de transição é um momento em que vacas leiteiras passam por grandes mudanças adaptativas (DRACKLEY, 2004). Esse período compreende três semanas antes até três semanas após o parto. Segundo Bell (1995), a maior demanda de nutrientes pelo feto e placenta ocorre nas três últimas semanas de gestação e o CMS pode cair 10\% a 30\% comparado com o início do período seco. A queda de progesterona e o aumento de estrógeno próximo ao parto pode ser um fator que contribui para a diminuição do CMS, porém, este mecanismo ainda não está muito bem esclarecido (GRUMMER, 1993; DRACKLEY et al., 2005).

Após o parto, com o início da lactação e com o rápido aumento da produção leiteira, aumenta a demanda por glicose, em um período em que o animal se encontra com um baixo CMS. As exigências líquidas para lactação praticamente dobram no período pré-parto para o pósparto como descrito na tabela 1. Segundo Overton et al. (1998), a necessidade diária de glicose de uma vaca holandesa é de 1000 a 1100 g/dia no final da gestação e de 2500 g/dia aos 21 dias após o parto. Devido ao baixo CMS a produção de propionato é insuficiente para sintetizar a quantidade de glicose necessária (DRACKLEY, 2004). Assim, aminoácidos da dieta e o catabolismo dos músculos esqueléticos juntamente com o glicerol proveniente da mobilização do tecido adiposo fornecem o restante da glicose necessária (DRACKLEY, 2005). 
Tabela 1. Exigências de energia líquida para lactação $(\mathrm{ELl})(\mathrm{MJ} / \mathrm{d})$ de vacas leiteiras e novilhas dois dias $(-2 \mathrm{~d})$ antes do parto e dois dias $(+2 \mathrm{~d})$ após o parto.

\begin{tabular}{lcccc}
\hline & \multicolumn{2}{c}{ Multípara $725 \mathrm{~kg}$} & \multicolumn{2}{c}{ Primípara $570 \mathrm{~kg}$} \\
\hline Função & $-2 \mathrm{~d}$ & $+2 \mathrm{~d}$ & $-2 \mathrm{~d}$ & $+2 \mathrm{~d}$ \\
\hline Manutenção & 46,9 & 42,2 & 38,9 & 35,6 \\
Gestação & 13,8 & --- & 11,7 & --- \\
Crescimento & --- & --- & 7,9 & 7,1 \\
Lactação & --- & 78,2 & -- & 62,3 \\
\hline Total & $\mathbf{6 0 , 7}$ & $\mathbf{1 2 0 , 4}$ & $\mathbf{5 8 , 5}$ & $\mathbf{1 0 5 , 0}$ \\
\hline
\end{tabular}

Calculado pelo National Research Council (2001), assumindo uma produção de leite com 4\% de gordura e $25 \mathrm{~kg} / \mathrm{d}$ para vacas multíparas e $20 \mathrm{~kg} / \mathrm{d}$ para vacas primíparas. Adaptado de Drackley et al. (2005).

O BEN nas primeiras semanas de lactação ocorre frequentemente em vacas leiteiras de alta produção, mesmo hígidas (RADOSTISTS et al., 2000). Para suprir o BEN ocorre à mobilização de ácidos graxos de cadeia longa dos triglicérides (TG) provenientes do tecido adiposo e esta mobilização acontece devido à alta relação hormônio do crescimento/insulina no sangue de vacas recém-paridas. Os TG liberados do tecido adiposo perdem sua molécula éster para facilitar seu transporte pela corrente sanguínea. A forma circulante dos TG é denominada AGNE (BEZERRA et al., 2014), que é a principal fonte de energia no início da lactação. A concentração de AGNE reflete a mobilização de tecido adiposo sendo proporcional ao grau de BEN (DRACLKLEY, 2005).

O fígado é o responsável pelo metabolismo dos AGNE no organismo, os quais podem ser completamente oxidados a dióxido de carbono para fornecer energia ao fígado que, além de utilizar os AGNE como fonte de energia, pode oxidar parcialmente os AGNE para produção de corpos cetônicos (acetato, acetoacetato e BHBA) que são liberados na corrente sanguínea para serem utilizados como fonte de energia por diversos tecidos, principalmente em situações de inanição e BEN (DRACKLEY, 2004). O fígado também possui a capacidade de reesterificar AGNE a TG que ainda nele acumulados ou secretados para utilização pela glândula mamária, coração e músculos esqueléticos. A aproximação do parto aumenta a habilidade do fígado em esterificar AGNE em TG. O máximo acúmulo de TG pelo tecido hepático ocorre entre o sétimo e décimo quarto dia de lactação (SEJRSEN; HVELPLUND; NIELSEM, 2006).

O metabolismo dos AGNE possui um papel importante na manutenção da homeostase no início da lactação. Entretanto, um excessivo acúmulo de TG no fígado devido à absorção de AGNE pode predispor a ocorrência de fígado gorduroso (BOBE et al., 2004). O BEN em conjunto com a limitada reserva de carboidratos no fígado após o parto pode aumentar a produção de corpos cetônicos. O BEN, a intensa mobilização de TG proveniente o tecido adiposo 
e a cetogênese são fatores predisponentes para afecções durante periparto em vacas leiteiras (DACKLEY et. al., 2005).

A cetose é uma doença metabólica comum em vacas leiteiras de alta produção no período pós-parto devido o BEN e a mobilização de tecido adiposo no início da lactação. A enfermidade se caracteriza pelo aumento nos níveis de corpos cetônicos no sangue, urina, leite e outros fluídos corporais em consequência ao aumento de AGNE e hipoglicemia. Esta desordem metabólica geralmente ocorre entre a primeira e a oitava semana de lactação (SOUZA, 2003).

Os AGNE quando atingem o fígado são beta-oxidados à acetil-CoA que podem ser completamente oxidados no ciclo de Krebs, parcialmente oxidados em corpos cetônicos ou reesterificados em TG. A capacidade de oxidação do ciclo de Krebs é dependente da disponibilidade de oxaloacetato gerado a partir do propionato, lactato e piruvato. Durante o BEN, a demanda por glicose aumenta e o suprimento de propionato diminui o que leva a desvio de oxaloacetato para a gliconeogênese retardando o ciclo de Krebs e o uso de acetil-CoA. Quando a disponibilidade de acetil-CoA excede a capacidade de oxidação pelo ciclo de Krebs, juntamente com a oxidação parcial de AGNE em acetil-CoA pelo fígado, ocorre um aumento na produção de corpos cetônicos e TG, predispondo a ocorrência de cetose e fígado gorduroso. Embora os corpos cetônicos possam ser usados como fonte de energia por diversos tecidos, o seu excesso no sangue pode ter efeitos negativos na saúde e produtividade dos animais (WHITE, 2015).

A cetose pode ser classificada em primária, secundária, alimentar por inanição ou por deficiência nutricional específica. A cetose primária ocorre em animais de alta produção, com boa condição corporal ,que recebem alimentação de boa qualidade, sendo o BEN a principal causa do desenvolvimento da doença. A cetose secundária é provocada por doenças como deslocamento de abomaso, retículo pericardite traumática, metrite, mastite ou outras doenças que diminuem a ingestão de alimentos. Por outro lado, cetose alimentar se desenvolve devido à alta ingestão de butirato, prercursor cetogênico, na silagem. A cetose por inanição acontece em animais com baixa condição corporal que recebem dietas de péssima qualidade, e a cetose por deficiência nutricional ocorre na carência de cobalto e fósforo devido a queda na ingestão de alimentos e insuficiente metabolização de propionato dentro do ciclo do ácido tricarboxílico (RADOSTITS et al., 2000).

Além da classificação quanto à origem, a cetose pode ser classificada em clínica e subclínica. No quadro clínico, o animal apresenta perda de peso e condição corpórea, queda na produção leiteira, fezes secas, hálito cetônico, hipoglicemia, acetonemia e cetonúria. Em casos mais severos, podem ocorrer sinais neurológicos como marcha em círculos, afastamento ou cruzamento das pernas, compressão da cabeça contra objetos, cegueira, lambedura da pele e 
objetos, apetite depravado, mastigação excessiva e salivação. No quadro subclínico da doença, o animal não apresenta sinais clínicos aparentes, exceto pela queda na produção leiteira, hipoglicemia e acetonemia (RADOSTITS et al., 2000; GARCIA, 2010).

O diagnóstico é realizado por meio dos sinais clínicos, epidemiologia e mensuração da concentração de BHBA no sangue, urina ou leite. $\mathrm{O}$ teste de urina possui dois inconvenientes, um é a dificuldade na coleta de urina que pode ser coletada de forma espontânea ou realizando massagem na região do períneo, porém em um estudo realizado por Osborne (2002), apenas 64\% dos animais testados tiveram sucesso na coleta por estímulo. Outro inconveniente do teste de urina é que a tira deve ser mergulhada na urina exatamente por 15 segundos, caso fique por um tempo maior o resultado pode ser falso positivo. O teste do leite leva vantagem sobre o de urina pela facilidade de coleta do material (OETZEL, 2007). O teste de BHBA no sangue total possui uma maior especificidade e sensibilidade quando comparado com os testes no leite e urina (OETZEL, 2004). Iwersen et al. (2009), concluíram que o medidor eletrônico de BHBA e glicose, é uma ótima ferramenta para diagnóstico de cetose utilizando sangue. Animais que apresentam $\mathrm{BHBA} \geq 3 \mathrm{mmol} / \mathrm{L}$ são diagnosticados com cetose clínica e BHBA entre 1,2mmol/L a 2,9mmol/L, com cetose subclínica (WHITE, 2015).

O principal objetivo do tratamento da cetose é reestabelecer a glicemia,por tanto, protocolos são descritos (tabela 2) para cetose primária. O fornecimento de propileno-glicol via oral (VO), administração de glicose intravenosa (IV), injeções intramusculares (IM) de glicocorticóides e insulina tem sido os principais tratamentos preconizados para o tratamento da cetose. O propileno-glicol, quando ingerido, pode ser transformado em propionato ou ser diretamente absorvido e utilizado como substrato para gliconeogênese, que resulta em aumento da glicose e insulina sanguíneas e queda na concentração de AGNE e BHBA (LOMANDER et al., 2012; FONSECA, 2015). A administração de glicose 50\% IV promove um aumento de até oito vezes os níveis de glicose sanguínea imediatamente após a infusão, porém, após 2 horas, os níveis de glicose retornam ao normal. Esta administração também promove uma diminuição na concentração de BHBA durante 24 horas (FONSECA, 2015). A injeção de glicocorticóides promove uma diminuição na produção de leite e consequentemente um aumento nos níveis sanguíneos de glicose em virtude da queda na utilização de glicose para sintetizar lactose, além disso, os glicocorticóides também estimulam a gliconeogênese (SOUZA, 2003). O uso da insulina, via subcutânea, diminui a lipólise e aumenta a lipogênese pelos seus efeitos anabólicos; com isso, ocorre maior utilização de corpos cetônicos como fonte de energia (FONSECA, 2015). 
Tabela 2. Principais produtos utilizados nos protocolos de tratamento para cetose

\begin{tabular}{lll}
\hline Produto & Via de administração & Dosagem \\
\hline Propileno-glicol & VO & $225 \mathrm{~g}, \mathrm{BID}$ por 2 dias \\
Glicose 50\% & IV & $100 \mathrm{a} 500 \mathrm{ml}$, Dose única \\
Dexametasona & IM & $0,04 \mathrm{mg} / \mathrm{kg}$ Dose única \\
Insulina & SC & $200 \mathrm{UI}$, SID por dois dias \\
\hline
\end{tabular}

Fonte: Adaptado de Fonseca (2015)

No tratamento da cetose secundária além do tratamento descrito anteriormente para a cetose primária, é importante eliminar o fator que está desencadeando a enfermidade primária concomitante. No caso da cetose alimentar devido a ingestão de altos níveis de butirato na silagem, a dieta deve ser manipulada a fim de diluir ou eliminar a quantidade desse AVG (FONSECA, 2015).

A principal forma de prevenção da cetose em bovinos e outras doenças do periparto inclui nutrição e manejo adequados (AROEIRA, 1998). Segundo Neto et al. (2011), o controle do escore corporal (ECC) em vacas leiteiras é uma ótima ferramenta na prevenção da cetose, visto que vacas obesas estão mais propensas em desenvolver este transtorno metabólico. O ECC em vacas leiteiras é avaliado em uma escala de 1 a 5 , sendo 1 o animal caquético e 5 o animal obeso (SOUZA, 2003). Ferguson e Otto (1989) recomendam um escore de 3,5 como ótimo no momento do parto.

Aditivos alimentares podem ser usados na dieta para prevenção da cetose, tais como a niacina, propionato de cálcio, propionato de sódio, propileno-glicol e colina. Estes suplementos para serem efetivos devem ser adicionados à dieta 2 a 3 semanas antes do parto (KAHN; LINE, 2010).

O uso da monensina sódica na dieta reduz a ocorrência de cetose devido a alteração na produção de ácidos graxos voláteis no rumem. Esse ionóforo aumenta a produção de ácido propiônico, prercursor glicogênico, e diminui a produção de ácido butírico, prercursor cetogênico, que leva a aumento da glicose disponível (SOUZA, 2003).

\section{CONSIDERAÇÕES FINAIS}

Vacas leiteiras durante o período de transição são mais susceptíveis à cetose pelo aumento da quebra de gordura corporal em consequência do BEN em que esses animais se encontram. $\mathrm{O}$ correto diagnóstico e tratamento da cetose é de suma importância para minimizar prejuízos econômicos e a debilidade na saúde do rebanho. O uso do medidor eletrônico de BHBA de uso 
humano pode ser uma excelente ferramenta para o diagnóstico de cetose. Porém, a prevenção ainda deve ser considerada para evitar prejuízos econômicos e metabólicos.

\section{REFERÊNCIAS}

ARAÚJO, D. B.. Monitoramento de doenças metabólicas no periparto - Parte I: Cetose subclínica. Milkpoint. 10 abr. 2013. Disponível em: http://www.milkpoint.com.br/radartecnico/medicina-da-producao/monitoramento-de-doencas-metabolicas-no-periparto-parte-icetose-subclinica-83317n.aspx. Acesso em: 15 abr. 2015.

AROEIRA, L. J. M. Cetose e infiltração gordurosa no fígado em vacas leiteiras. Juiz de Fora: EMBRAPA-CNPGL, 1998. 23p.

BACILA, M.. Bioquímica veterinária. 2. ed. São Paulo: Robe, 2003.

BELL, A. W. Regulation of organic nutrient metabolism during transition from late pregnancy to early lactation. Journal of Animal Science. v.73. p.2804-2819. 1995

BEZERRA, et al. Major metabolic diseases affecting cows in transition period. International Journal of Biology. v.6, n.3, 2014.

BOBE, G., YOUNG. J. W., BEITZ D. C. Pathology, etiology, prevention, and treatment of fatty liver in dairy cows. Jornal of Dairy Science. v.87, p.3105-3124, 2004.

CUNNINGHAM, J. G. Tratado de fisiologia veterinária. 3.ed. Rio de Janeiro: Guanabara Koogan, 2004. 579p.

DIVERS, T. J.; S. F. PEEK. Rebhun's Diseases of Dairy Cattle. 2.ed. St. Louis: Elsevier, 2008. 686p.

DRACKLEY, J. K. et al. Physiological and pathological adaptations in dairy cows that may increase susceptibility to periparturient diseases and disorders. Italian Journal of Animal Science. v.4, p.323-344, 2005.

DRACKLEY, J.K. Physiological adaptations in transition dairy cows. In: Proc. Minnesota Dairy Herd Health Conf... Saint Paul, 2004. 74-87p.

DUFFIELD, T.F. Subclinical ketosis in lactating dairy cattle: metabolic disorders of ruminants. Veterinary Clinics: Food Animal Practice. v.16, p231-53, 2000.

FERGUSON, J. D.; OTTO, K. A. Managing body condition in dairy cows. In: CORNELL NUTRITION CONFERENCE FOR FEED MANUFACTURERS, 1989, Ithaca. Anais... Ithaca, 1989 , p.75-87.

FONSECA, A.P.S. Cetose: diagnóstico e tratamento: revisão bibliográfica. 2015. Dissertação (Mestrado em veterinária). Universidade do Porto. 
GARCIA, A.M.B. Avaliação metabólica de vacas leiteiras submetidas a diferentes estratégias de prevenção do balanço energético negativo no pós-parto. 2010. Dissertação (Mestrado em veterinária). Faculdade de Veterinária. Universidade Federal do Rio Grande do Sul. Porto Alegre.

GRUMMER, R. R. Etiology of lipid-related metabolic disorders in periparturient dairy cows. Journal of Dairy Science. v.76, p.3882-3896. 1993.

HERDT, T.H., 2000. Ruminant adaptation to negative energy balance. Influences on the etiology of ketosis and fatty liver. Veterinary Clinics of North America: Food Animal Practice. v.16, p.215-230, 2000.

KAHN, CM; Line S., 2010. The Merck Veterinary Manual (10th). Merck Sharp \& Dohme Corp., a subsidiary of Merck \& Co. Inc., Whitehouse Station, NJ.

LOMANDER, H. et al. Supplemental feeding with glycerol or propylene glycol of dairy cows in early lactation - Effects on metabolic status, body condition, and milk yield. Journal of Dairy Science. v.95, p.2397-2408. 2012.

OETZEL, G.R. Monitoring and testing dairy herds for metabolic disease. Veterinary Clinics: Food Animal Practice. v.20, p. 651-674, 2004.

OETZEL G.R. Understanding the Impact of Subclinical Ketosis. In: AMERICAN ASSOCIATION OF BOVINE PRACTITIONERS, 2007, UW-Madison. Conf... Vancouver, 2007.

OVERTON, T.R. et al. Hepatic gluconeogenesis and whole-body protein metabolism of periparturient dairy cows as affected by source of energy and intake of the prepartum diet. Journal of Dairy Science, v.81, p.295, 1998.

RADOSTITS, O.M. et al. Clínica veterinária: um tratado de doenças dos bovinos, ovinos, suínos, caprinos e equinos. 9. ed. Rio de Janeiro: Guanabara Koogan, 2000. 1737p.

SEJRSEN, K.; HVELPLUND, T.; NIELSEM, M.O. Ruminant physiology: digestion, metabolism and impact of nutrition on gene expression, immunology and stress.

Wageningen, Netherlands: Wageningen Academic Publishers. p.600, 2006.

SOUZA, A. N. M. Cetose dos bovinos e lipidose hepática. 2003. 18 f. Seminário (Bioquímica do Tecido Animal). Universidade Federal do Rio Grande do Sul - Programa de Pós-Graduação em Ciências Veterinárias. Porto Alegre.

VICENTE, F. et al. Subclinical Ketosis On Dairy Cows in Transition Period in Farms With Contrasting Butyric Acid Contents in Silages. Hindawi Publishing Corporation, 2014.

WHITE, H.M. The Role of TCA Cycle Anaplerosis in Ketosis and Fatty Liver in Periparturient Dairy Cows. Animals, v. 5, p.793-802, 2015. 
